\title{
Designing clinical trials in paediatric inflammatory bowel diseases: a PIBDnet commentary
}

\author{
Dan Turner, ${ }_{1}^{1}$ Anne M Griffiths, ${ }^{2}$ David Wilson, ${ }^{3}$ Diane R Mould, ${ }^{4}$ Robert N Baldassano, ${ }^{5}$ \\ Richard K Russell, ${ }^{6}$ Marla Dubinsky, ${ }^{7}$ Melvin B Heyman, ${ }^{8}$ Lissy de Ridder, ${ }^{9}$ \\ Jeffrey Hyams, ${ }^{10}$ Javier Martin de Carpi, ${ }^{11}$ Laurie Conklin, ${ }^{12,13}$ William A Faubion, ${ }^{14}$ \\ Sibylle Koletzko, ${ }^{15}$ Athos Bousvaros, ${ }^{16}$ Frank M Ruemmele (D) ${ }^{17,18,19}$
}

For numbered affiliations see end of article.

\section{Correspondence to}

Professor Frank M Ruemmele, Hôpital Necker Enfants Malades, Paris 75015, France: frank.ruemmele@nck.aphp.fr

Received 27 November 2018 Revised 14 February 2019 Accepted 19 March 2019 Published Online First 12 April 2019

\section{Check for updates}

(c) Author(s) (or their employer(s)) 2020. No commercial re-use. See rights and permissions. Published by BMJ.

To cite: Turner $\mathrm{D}$, Griffiths AM, Wilson D, et al. Gut 2020;69:32-41.

\begin{abstract}
Introduction The optimal trial design for assessing novel therapies in paediatric IBD (PIBD) is a subject of intense ongoing global discussions and debate among the different stakeholders. However, there is a consensus that the current situation in which most medications used in children with IBD are prescribed as off-label without sufficient paediatric data is unacceptable. Shortening the time lag between adult and paediatric approval of drugs is of the upmost importance. In this position paper we aimed to provide guidance from the global clinical research network (Pediatric Inflammatory Bowel Disease Network, PIBDnet) for designing clinical trials in PIBD in order to facilitate drug approval for children.

Methods A writing group has been established by PIBDnet and topics were assigned to different members. After an iterative process of revisions among the writing group and one face-to-face meeting, all statements have reached consensus of $>80 \%$ as defined a priori. Next, all core members of PIBDnet voted on the statements, reaching consensus of $>80 \%$ on all statements.

Comments from the members were incorporated in the text.

Results The commentary includes 18 statements for guiding data extrapolation from adults, eligibility criteria to PIBD trials, use of placebo, dosing, endpoints and recommendations for feasible trials. Controversial issues have been highlighted in the text.

Conclusion The viewpoints expressed in this paper could assist planning clinical trials in PIBD which are both of high quality and ethical, while remaining pragmatic
\end{abstract}

\section{INTRODUCTION}

The timing of drug studies in paediatric IBD (PIBD) has been suboptimal with most being conducted long after approval has been granted for adult IBD (figure 1). This time lag leads to extensive off-label use of drugs in children, often without clear guidance on appropriate dosing. This is of concern since paediatric dosing of biologics, extrapolated from studies in adults, has been often shown eventually to be too low (table 1 summarises a subset of the important relevant randomised paediatric studies). ${ }^{1-3}$

Clinical trials conducted in children must balance quality with feasibility, while considering

\section{Significance of this study}

What is already known on this subject?

- Children/adolescents with IBD have often a particularly active disease requiring early biologic therapy, however access to new medication is often delayed compared with adult patients.

- Clinical drug trials are more difficult to perform in children compared with adult patients with IBD.

- A simple copy/paste approach from randomised controlled trial (RCT) in adult patients with IBD to children with IBD is not appropriate.

What are the new findings?

- This is the first consensus process of paediatric IBD (PIBD) experts on how to facilitate/optimise clinical drug trial designs for children with IBD.

- Extrapolation from adult trials together with pharmacokinetic/pharmacodynamic studies and safety data might suffice for drugs of a class with existing approval for PIBD.

- Particular attention should be drawn to dosing studies for younger children $(<30 \mathrm{~kg})$ since they may require higher $\mathrm{mg} / \mathrm{kg}$ dosing compared with older children and adults.

- Feasibility (adapted small sample size, reduced number of invasive procedures, reduced washout periods prior to inclusion) is a major criterion for a successful RCT.

the unique age-specific ethical considerations. This balance is much more challenging than in adult trials, and thus a 'copy-paste' approach from adult protocols is often inappropriate. Parents must make all decisions in the best interest of their children and cannot consent on their child's behalf to altruistically participate in interventional clinical studies. This ethos is the basis for the notion that paediatric trials must be designed in a way that provides potential for direct benefit to the enrolled child. Enrolling children in clinical trials of drugs that are already widely used in adults is, therefore, particularly challenging, and a placebo-controlled design in this circumstance is most often untenable for many investigators 
Significance of this study

How might it impact on clinical practice in the foreseeable future?

- Avoidance of foreseeable unsuccessful RCT parameters (too many endoscopies, unrealistic large number of patients needed, and so on).

- New and easily attainable endpoints for RCTs combining objective measures as well as patient experienced symptoms.

- Improvement of global collaboration among PIBD experts as well as between PIBD experts, patient/parent organisations, agencies, Clinical Reasearch Organisations and/or pharmaceutical industry.

- Facilitating clinical drug trials while increasing the chances for faster access of children/adolescents with IBD to new medications.

and caregivers. Nevertheless, inclusion of children in studies of new therapies is vital, given the need to verify optimal dosing and monitor safety in children. This, especially in the youngest age groups who are increasingly affected by IBD and their response to drugs, often differs from adolescents. This review aims to highlight the age-specific challenges in regulatory paediatric trial design offering solutions to common pitfalls. These pitfalls are common and repeatedly discussed with pharmaceutical companies, regulatory agencies, the scientific community and families.

\section{METHODS}

Pediatric Inflammatory Bowel Disease Network (PIBDnet, www.pibd-net.org) is a network of PIBD experts over the world with particular interest and experience in clinical research, especially drug trials, for children with IBD. PIBDnet is actively conducting randomised controlled trial (RCT) in the field of PIBD. A steering committee formulated subheadings to be addressed in this position paper, each addressed by two to three authors and iteratively reviewed for revisions over emails and one face-to-face meeting. The paper was then sent to the core members of PIBDnet for revoting. A threshold for consensus was determined a priori as $>80 \%$ which has been

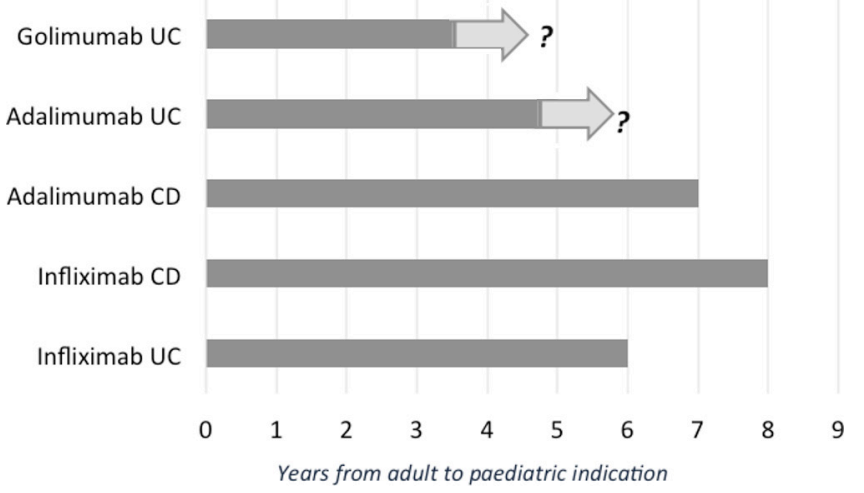

Figure 1 Years' interval from approval of biologics in adults to approval in children (top two have not received pediatric indication yet and paediatric trials are still ongoing) (Reprinted with permission from Turner D, Koletzko S, Griffiths AM, et al. Use of placebo in pediatric inflammatory bowel diseases: a position paper from ESPGHAN, ECCO, PIBDnet, and the Canadian Children IBD Network. J Pediatr Gastroenterol Nutr 2016;62:183-7[14]). CD, Crohn's disease. achieved after the first round of voting (total of 29 voting experts: 26 core members of PIBDnet and 3 external experts in the authors; online supplementary appendix 1). Comments obtained during the voting process were incorporated when possible in the text.

\section{DATA EXTRAPOLATION TO SUPPORT PAEDIATRIC LABELLING IN IBD \\ Statements}

1. Building on prior adult RCTs, pharmacokinetic/pharmacodynamic (PK/PD) paediatric data should suffice to approve drugs that are not of new category (ie, availability of another approved drug in children from the same class), on condition that PK/PD (ie, exposure response) relationship is similar to adults $^{4}$ (97\% agreement).

2. Dosing and safety, however, must be demonstrated independently from adult studies and cannot be extrapolated (100\% agreement).

Extrapolating efficacy from adults to children was first proposed by the Food and Drug Administration (FDA) in the 1994 Pediatric Labeling Rule. FDA guidance articulates the path to three regulatory outcomes: no extrapolation, full extrapolation and partial extrapolation (https://www.fda.gov/ downloads/drugs/guidances/ucm425885.pdf). Of 166 products approved for paediatrics between 1998 and 2008, twenty-four relied completely on extrapolated data and 113 used partial extrapolation. ${ }^{5}$ The path to paediatric labelling for the three FDA-approved IBD therapies (balsalazide, infliximab and adalimumab) involved partial extrapolation that assumes children have a similar disease course and response to intervention as adults but exposure response may differ. Subsequent efficacy trials in children would then be required if no PD measurements are available to predict efficacy. For partial extrapolation, one efficacy trial may be sufficient. In both cases, it is vital to start enrolling into the paediatric trial shortly after the results of the adult phase 3 trial are known, even prior to full publication. This will both ensure shortening the time to paediatric labelling and increase the feasibility of the trial (see below).

A key component for both partial and full extrapolation is prior dose selection that achieves an exposure range comparable to what has been observed in adults. PK/PD phase 2 trial designs measure the incremental benefit of increasing exposure (bioavailability) on drug response. Doses with exposures leading to mid-effect and high-effect sizes can be further assessed in a larger, controlled phase 3 study. We have learnt the importance of adequate exposure of biologics for maximising response in IBD (more important than dose), as the PK/PD of many monoclonal antibodies is not linear. Biosimilars in children may follow a different approval path, as detailed elsewhere. ${ }^{6}$ In all cases, whether following partial or full extrapolation, a clear plan for safety monitoring must to be in place, but this does not necessarily require an RCT.

\section{DOSING}

\section{Statements}

1. Dosing in paediatric trials should acknowledge that younger children (as a general rule $\sim<30 \mathrm{~kg}$ but this could differ) may require higher dose per kilogram than older children and adults $(97 \%$ agreement $)$.

2. Either body surface area (BSA)-based dosing or stratified perkilogram dosing (ie, different per-kilogram dose in different age groups) should be considered. In either case, PK/PD dose 


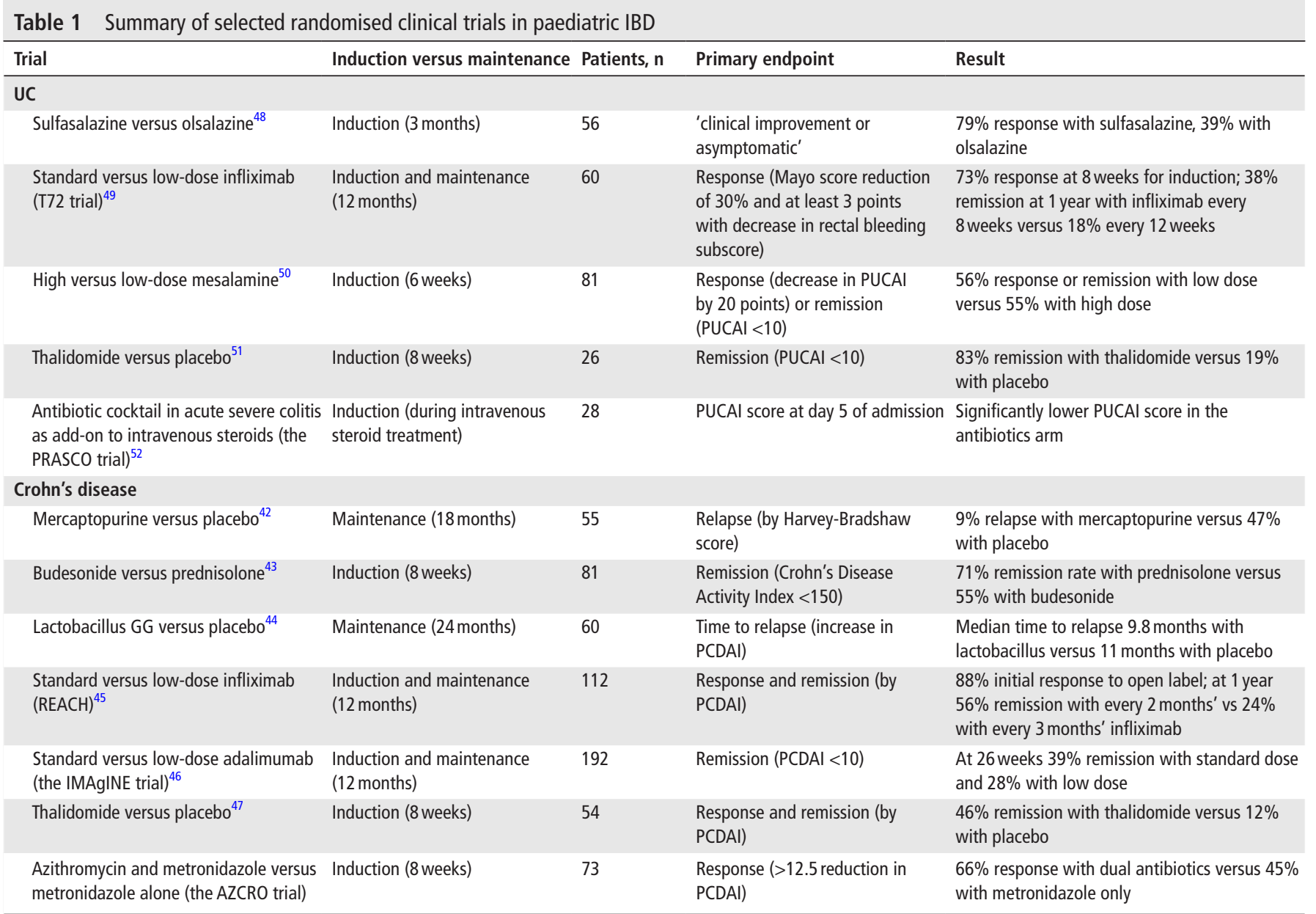

PCDAl, Pediatric Crohn's Disease Activity Index; PUCAI, Pediatric Ulcerative Colitis Activity Index.

ranging studies across all age groups are necessary, especially in the youngest (100\% agreement).

Many monoclonal antibody medications are dosed according to body weight (eg, $\mathrm{mg} / \mathrm{kg}$ ), despite weight either not predicting drug clearance or, more commonly, the relationship not being proportional (ie, the clearance reduction is not proportional to the reduction in weight). Dosing based on $\mathrm{mg} / \mathrm{kg}$ therefore frequently results in lower concentrations in smaller children, and this trend increases with lower body weight. ${ }^{7}$ For instance, weight-based dosing of steroids results in a lower drug level than BSA-based dosing and the difference decreases proportionally up to weights of $30 \mathrm{~kg}$; the same has been shown with infliximab, golimumab and adalimumab in PIBD. ${ }^{1-489}$ Simulated trough concentrations for the approved dose of infliximab are lower as weight decreases (figure 2). ${ }^{10}$ Similarly, median area under the curve (AUC) values in children aged 6-17 and 2-6years were, respectively, $20 \%$ and $40 \%$ lower than the predicated AUC for adults following administration of $5 \mathrm{mg} / \mathrm{kg}$ infliximab every 8 weeks. ${ }^{11}$ Poor planning of dosing in the paediatric clinical trials has led the European Crohn's and Colitis Organization (ECCO) and the European Society of Paediatric Gastroenterology, Hepatology and Nutrition (ESPGHAN) to recommend higher doses of adalimumab, infliximab and golimumab than those used in the paediatric trials. ${ }^{12}$ It is thus important to include children as young as 2 years in trials but excluding those with known monogenetic IBD-like disease.

Conversely, monoclonal antibody medications dosed based on BSA (eg, $\mathrm{mg} / \mathrm{m}^{2}$ ) may expose smaller children to higher relative doses than adult patients. ${ }^{13}$ Flat dosing (ie, 'one size fits all') results in overdosing in smaller patients and underdosing in bigger patients. For all three dose metrics $\left(\mathrm{mg} / \mathrm{kg}, \mathrm{mg} / \mathrm{m}^{2}\right.$ and flat dosing), the younger patients are exposed to a more significant underdosing or overdosing. An appropriately body size-based stratified approach may overcome these limitations.

\section{PLACEBO}

\section{Statements}

1. No child with IBD should be managed with a known inferior treatment than routinely available. Therefore, the use of placebo should be avoided in studies where the study drug has previously been shown to be superior to placebo in children and/or in adults (93\% agreement).

2. For a new drug category, placebo should only be used in children who have exhausted other approved therapies in paediatrics, in order to ensure genuine equipoise between available active treatments and placebo both within the clinical trial and outside (as part of clinical practice) (83\% agreement).

3. If true equipoise exists (both within and outside the trial) placebo may be considered only when complete remission has been achieved after (open-label) induction therapy; 'response' without remission is insufficient. Very early escape points should be planned to allow prompt treatment for children whose disease has become active ( $83 \%$ agreement).

4. Adolescents may be included in adult placebo-controlled trials on condition that the relevant standard treatments 


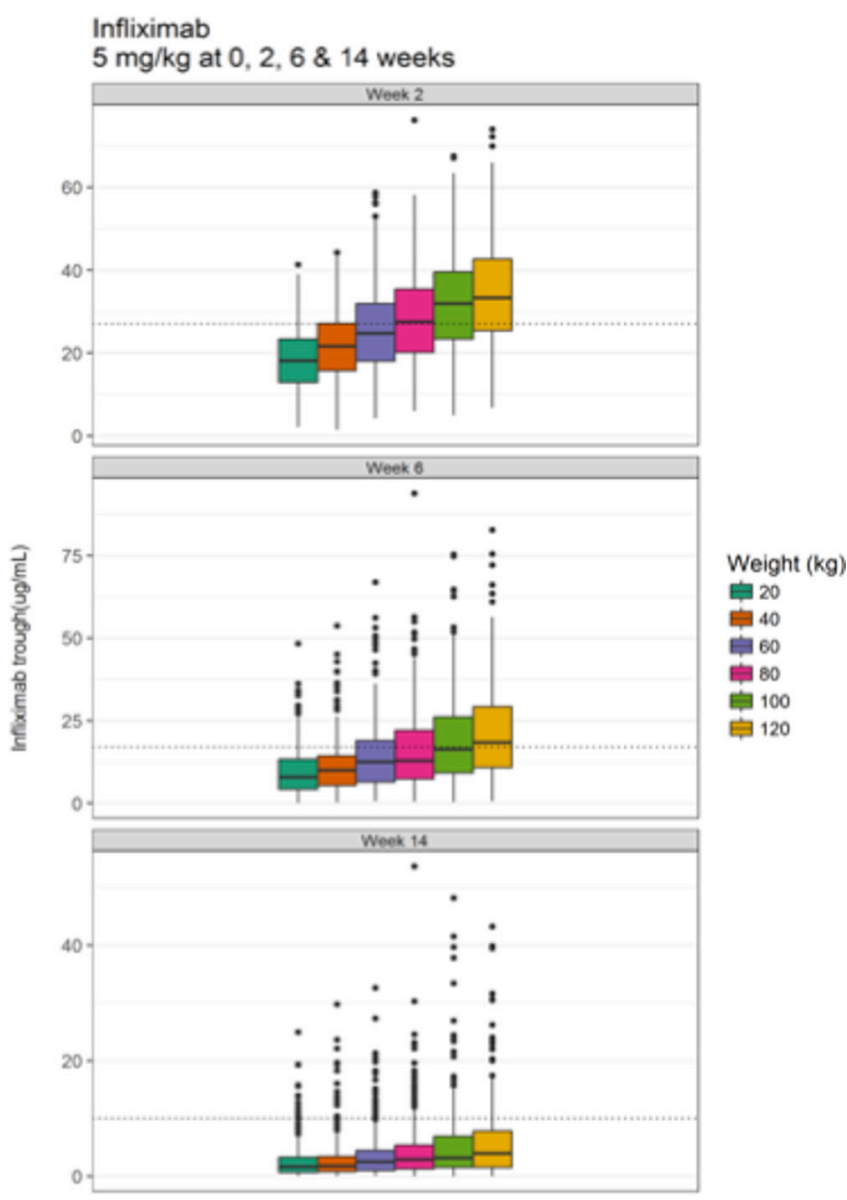

Figure 2 Simulated ranges of infliximab trough concentrations following administration of $5 \mathrm{mg} / \mathrm{kg}$ at weeks 0,2 and 6 . The boxes are stratified in $20 \mathrm{~kg}$ weight increments.

outside the trials have been exhausted to ensure equipoise of the placebo arm with the standard of care. A sufficient sample size of the adolescent subgroup must be ensured to allow meaningful conclusions for this age group (86\% agreement).

The controversy of including placebo in PIBD trials has been extensively reviewed in a position paper from ESPGHAN, ECCO, PIBDnet and the Canadian Children IBD network, ${ }^{14}$ and thus only brief guiding rules are summarised herein. Placebo is easier to justify in trials of new drug classes after all paediatricapproved medications have failed. Paediatric and adult IBDs are so similar in terms of treatment responsiveness that therapies shown to be effective in adults cannot be considered in equipoise with placebo in children. In the same way, equipoise regarding efficacy of a particular drug is diluted after extensive off-label use. Thus industry-sponsored trials may become more ethically challenging when a time gap exists between the adult and paediatric studies. The tendency to move away from clinical outcomes to objective measures of mucosal healing also decreases dependence on placebo to prove drug efficacy, since complete mucosal healing is rarely seen with placebo treatment ( $\sim$ 0\%-10\% depending on the trial).

Several scenarios can be discussed for PIBD trials, particularly for new medicines:

Scenario 1: Generally, placebo should only be used if the child is in remission and there is a genuine equipoise between the active treatment and placebo (table 2). This means that, in contrast to adults, after an open-label induction phase only remitters and not responders should be considered for randomisation to
Table 2 Circumstances that placebo may be incorporated in paediatric IBD trials

As an add-on treatment to an effective treatment. Medications commenced prior to the trial and allowed to be continued after randomisation cannot usually be

Add-on to an effective treatment considered 'effective' since they have typically failed by the virtue of the need to start the study drug.

No prior adult trials In trials of drugs without prior adult approval so there is equipoise about the efficacy of the product under investigation; the use of placebo may be justified if all approved medical treatments have been exhausted and the child is in remission at the time of randomisation to placebo following open-label induction. Complete clinical remission should be the minimum standard. This may be used in a randomised withdrawal trial in which children are randomised into placebo following several months of treatment with an effective drug (rather than few weeks' induction).

No other valid alternatives exist

In rare circumstances placebo may be considered in active patients, such as when no other approved or offlabel alternative drugs are available and the patient is in stable non-severe condition.

placebo or investigational drug when otherwise justified. There is no agreement on the definition of remission for this purpose but clearly complete clinical remission is the minimum standard. Well-designed and rapid escape strategies are most important in this scenario, allowing prompt treatment for children whose disease has become active. It is unacceptable to make children with active IBD wait for rescue therapy. If a placebo-controlled RCT is based on repeat endoscopic evaluations to confirm active disease (see below), feasibility will be further compromised.

Scenario 2: Randomisation to different dosing schemes of the investigational drug without placebo is the most often selected design in PIBD trials. To obtain clinically meaningful results without the use of a placebo arm, objective measures of efficacy are indispensable, such as achievement of mucosal healing. However, repeated endoscopies are poorly accepted by children and their parents, compromising the feasibility of a trial. Objective non-invasive biomarkers of mucosal healing, such as faecal calprotectin, combined with limited number of endoscopic assessment as outlined below, markedly facilitate the realisation of an RCT. An excellent way to improve the significance of clinical measures as outcome parameters is to use prospective randomised open-blind endpoints, where the evaluating investigator is not aware of the allocated treatment arm.

Scenario 3: Recent study design in IBD includes adolescents in adult trials of drugs not yet approved. This design is encouraged as it ensures equipoise inside the trial and allows early paediatric labelling. However, children enrolled to such trials must have failed approved drugs available routinely to ensure equipoise also outside the trial. If this design is used, appropriate sample size of the adolescent subgroup must be ensured to provide sufficient data to draw conclusions. This option may be considered when there are no particular safety concerns and the study only includes children older than 12 years of age and weighing over $40 \mathrm{~kg}$. Waiving the need for placebo in the adolescents group could be more appealing, taking advantage of the fact that both groups follow the same protocol. Thus, it is reasonable to use the placebo effect from the young adults also for the adolescents. Incorporating adolescents into adult studies may shorten the time to paediatric adoption, and a smaller dose ranging study of younger children may later supplement the data. Although at times the investigational drug may seem more appealing or safer 
than routinely available treatments, this cannot be determined prior to the trial's results. The investigational treatment may be futile, as in the Mongersen phase 3 trial, and even harmful as in the case of Secukinumab. ${ }^{15}$

\section{ELIGIBILITY CRITERIA}

\section{Statements}

1. In UC, confirmation of baseline disease activity should include sigmoidoscopic evaluation especially for drugs of new category (using the Mayo Endoscopic Subscore or the Ulcerative Colitis Endoscopic Index of Severity [UCEIS]) (93\% agreement).

2. In Crohn's disease (CD), baseline disease activity should include ileocolonoscopy for drugs of new categories (using the Simple Endoscopic Score for Crohn's Disease, SES-CD). Otherwise, the Pediatric Crohn's Disease Activity Index (PCDAI) versions or patient-reported outcome (PRO) together with an objective measure of inflammation (eg, calprotectin or C-reactive protein [CRP]) could suffice $(83 \%$ agreement).

Narrow eligibility criteria regarding age, disease severity, disease location or previous treatments will present additional difficulties to recruitment of children. Too broad will lead to heterogeneity of included patients. In some studies, it might not be unreasonable to include children diagnosed with IBDunclassified (IBD-U), now that standard classification of this subgroup has been validated. ${ }^{16}$

Two scoring systems, the Pediatric Ulcerative Colitis Activity Index (PUCAI) and Mayo score, have been successfully used in previous clinical trials in paediatric UC. While only the PUCAI has been validated in children, the Mayo score allows intuitive comparison with adult trials. The PUCAI correlates well with endoscopic appearance of the colonic mucosa with a concordance of $\sim 80 \% .{ }^{17}$ For benchmarking the two scores, a PUCAI $<15$ points has been found to best reflect a Mayo scoredefined remission (ie, total Mayo score of $\leq 2$ points, with no subscore $>1$ point) with area under the receiver operating characteristic curve (AUROC) $=0.93$ (95\% CI 0.88 to 0.99), and a change in PUCAI of at least 20 points reflects a
Mayo score-defined response (ie, a decrease from baseline in the total Mayo score of $\geq 3$ points and $\geq 30 \%$, with a decrease in the subscore for rectal bleeding by $\geq 1$ point or an absolute subscore of 0 or 1 ) with AUROC $=0.97(95 \% \text { CI } 0.92 \text { to } 1.0)^{1718}$ (figure 3).

The European Medicines Agency (EMA) accepts using the PUCAI to screen patients with paediatric UC for trials and to grade disease activity into mild, moderate or severe (http://www. ema.europa.eu/docs/en_GB/document_library/Regulatory_and_ procedural_guideline/2016/01/WC500200026.pdf). However, in trials evaluating new categories of drugs, endoscopic activity should be determined prior to randomisation ensuring a Mayo Endoscopic Subscore of at least 2. Neither the Mayo score nor the UCEIS has been assessed for their psychometric properties in children. The former has the advantage of being used in practice in several paediatric trials and the latter that its development has followed a much more rigorous process. More studies on the psychometric properties of the UCEIS in children are required before making it the preferred endoscopic tool.

Assessment of disease activity in CD is more challenging because of the dissociation between clinical symptoms and mucosal inflammation. Nonetheless, complete ileocolonoscopy is feasible once or at most twice during paediatric trials (see the Endpoints section). Thus, the PCDAI ( $\geq 30$ points ${ }^{19}$ ) or weighted PCDAI (wPCDAI) ( $>40$ points ${ }^{20}$ ) may be used to select those with moderate disease at enrolment, or a MucosalInflammation Non-Invasive (MINI) index score $>8$ (composed of stool frequency, CRP, erythrocyte sedimentation rate [ESR] and faecal calprotectin) which has a high positive predictive value for active mucosal inflammation. ${ }^{21}$ If ileocolonoscopic assessment is performed, the adult SES-CD should be used.

\section{ENDPOINTS}

\section{Statements}

1. In general, endpoints should reflect control of mucosal or transmural inflammation. The choice of the outcome measure to reflect this concept should be individualised based on the balance of accuracy and feasibility (97\% agreement).
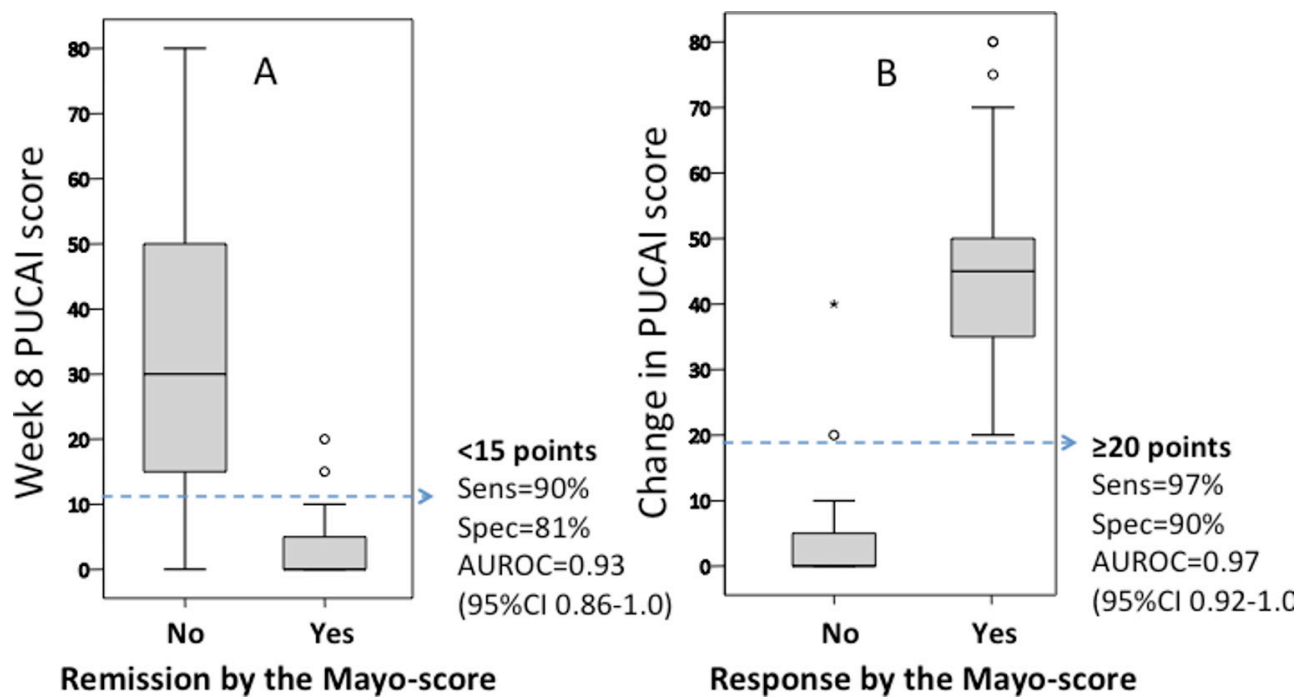

Figure 3 The cut-off values of the PUCAI that correspond best with remission (A) and response (B) defined by the Mayo score (Reprinted with permission from Turner D, Griffiths AM, Veerman G, et al. Endoscopic and clinical variables that predict sustained remission in children with ulcerative colitis treated with infliximab. Clin Gastroenterol Hepatol 2013;11:1460-5 [17]). AUROC, area under the receiver operating characteristic curve; PUCAI, Pediatric Ulcerative Colitis Activity Index. 
Table 3 Endpoints in paediatric clinical trials

\begin{tabular}{|c|c|c|c|c|c|c|}
\hline Type of clinical trial & Disease activity endpoint & $\begin{array}{l}\text { Clinical response } \\
\text { endpoint }\end{array}$ & $\begin{array}{l}\text { Linear growth and } \\
\text { bone formation }\end{array}$ & $\begin{array}{l}\text { Endoscopic endpoints } \\
\text { (remission; response) }\end{array}$ & $\begin{array}{l}\text { Patient-reported outcome } \\
\text { measure }\end{array}$ & HRQOL \\
\hline \multicolumn{7}{|l|}{ Crohn's disease } \\
\hline Short-term induction & $\begin{array}{l}\text { Steroid/EEN-free clinical remission defined } \\
\text { in table } 4\end{array}$ & $\begin{array}{l}\text { Drop in }>17.5 \text { by the } \\
\text { wPCDAl or }>12.5 \text { by the } \\
\text { PCDAl, or remission }\end{array}$ & Not applicable & $\begin{array}{l}\text { SES-CD } \\
\text { ( }<3 \text { points; }>50 \% \text { decrease) or } \\
\text { CDEIS }(<6 ;>50 \% \text { decrease) } \\
\text { MINI index in visits without } \\
\text { endoscopy }\end{array}$ & $\begin{array}{l}\text { TUMMY-CD when available } \\
\text { (until then use the stool and } \\
\text { abdominal pain items from } \\
\text { PCDAI) }\end{array}$ & IMPACT-III \\
\hline Long-term maintenance & $\begin{array}{l}\text { Sustained steroid-free remission: PCDAl or } \\
\text { wPCDAI remission at } \geq 2 \text { time points (eg, } 30 \\
\text { and } 54 \text { months) }\end{array}$ & Not applicable & $\begin{array}{l}\text { Height velocity } \\
\text { (cm/year); serum/ } \\
\text { urine markers of bone } \\
\text { formation* }\end{array}$ & As above & As above & As above \\
\hline \multicolumn{7}{|l|}{ UC } \\
\hline Short-term induction & $\begin{array}{l}\text { Steroid-free clinical remission, defined in } \\
\text { table } 4\end{array}$ & $\begin{array}{l}\text { Drop of }>20 \text { points by } \\
\text { the PUCAl, or remission }\end{array}$ & Not applicable & $\begin{array}{l}\text { Mayo Endoscopic Subscores } \\
\text { of } 0-3 \\
\text { Remission: subscore }=0\end{array}$ & TUMMY-UC & IMPACT-III \\
\hline Long-term maintenance & $\begin{array}{l}\text { Sustained steroid- free remission at } \geq 2 \text { time } \\
\text { points (eg, } 30 \text { and } 54 \text { months) }\end{array}$ & Not applicable & Not applicablet & As above & As above & As above \\
\hline
\end{tabular}

*Dual energy $\mathrm{X}$-ray absorptiometry (DEXA) scan is not responsive enough to observe a meaningful change over a year.

tGrowth is rarely impaired in UC in the range of $~ 5 \%$, and osteopenia is less common than in Crohn's disease.

CDEIS, Crohn's Disease Endoscopic Index of Severity; EEN, exclusive enteral nutrition; HRQOL, health-related quality of life; MINI, Mucosal-Inflammation Non-Invasive; PCDAI, Pediatric Crohn's Disease Activity

Index; PUCAI, Pediatric Ulcerative Colitis Activity Index; SES-CD, Simple Endoscopic Score for Crohn's Disease; wPCDAl, weighted PCDAI.

2. While the ideal trial design includes three endoscopic evaluations (prior to study entry, at the end of the induction phase and at the end of the maintenance phase), in children, two, one and even no evaluations may be optional based on the study design, whether the drug represents a new category and the availability of prior supportive data. While three sigmoidoscopic evaluations may be feasible (eg, in a UC trial of a drug of new category), performing three ileocolonoscopies in 1 year (as would be required in CD) is too burdensome in children. Clinical endpoints should thus be used at the end of the induction period in $\mathrm{CD}$ if participants continue to a maintenance phase $(83 \%$ agreement $)$.

3. In some trials endoscopic evaluations performed as part of clinical practice up to 1 month prior to screening may be acceptable at baseline if treatment has been stable (providing that photos or videos are available for confirming the results) $(100 \%$ agreement $)$.

4. MR enterography (MRE) (in children $>6$ years of age) or bowel ultrasound should supplement colonoscopies in CD to capture transmural healing rate and location of disease outreaching endoscopy. Radiological measures can also be used for imputing SES-CD data when ileal intubation has been unsuccessful (100\% agreement).

5. Steroid and exclusive enteral nutrition (EEN)-free remission whether clinically (ie, disease activity indices or PRO) or endoscopically should be the preferred endpoint, measured at more than one time point (ie, sustained remission). PROs should only be used in adjunct together with an objective measure of inflammation (eg, CRP and calprotectin) (97\% agreement).
6. Disease activity should be captured at every visit via PCDAI or wPCDAI in CD and PUCAI in UC. The development of PROs should be facilitated in PIBD (97\% agreement).

7. When endoscopic assessment is waived, calprotectin (eg, level $<200-300$ ) should accompany clinical remission (in UC PUCAI $<10$, and in CD either wPCDAI $<12.5$ or a composite of PCDAI $<10$ or $<7.5$ without the height item) (tables 3 and 4) (90\% agreement).

The reader is referred to a detailed position paper from the paediatric committee of ECCO on selecting endpoint measures in PIBD trials. ${ }^{22}$ Recent developments in IBD have resulted in moving away from symptom-based scoring to more objective measures of inflammation, such as endoscopic appearance, inflammatory biomarkers and radiological endpoints. However, the choice of the endpoint must carefully balance the desire to incorporate the perfect scientific endpoint (eg, several ileocolonoscopies) with the understanding that children are much more sensitive to repeated invasive procedures and that, pragmatically, they are more difficult to organise (eg, needing general anaesthesia).

The choice of number of endoscopic assessments (none to three) should be based on the type of the study and drug under evaluation: prior to study entry, at the end of the induction phase, at the end of the maintenance phase, or less. In addition, the US FDA now requires a measurement of a PRO as a treatment endpoint in IBD trials. PROs capture symptoms important to and reported directly by patients (or by an observer in children younger than 8 years of age), without interpretation by a physician. A PRO measure is distinct from disease activity indices (eg, PCDAI and PUCAI) and from health-related quality of life

Table 4 Cut-off points of disease activity indices in paediatric IBD

\begin{tabular}{|c|c|c|c|c|c|}
\hline & Response & Remission & Mild disease & Moderate disease & Severe disease \\
\hline PCDAI & Drop of $>12.5$ points & $\begin{array}{l}<10 \text { points or }<7.5 \text { without the } \\
\text { height item* }\end{array}$ & 10-27.5 points & $30-37.5$ points & 40-100 points \\
\hline wPCDAl & Drop of $\geq 17.5$ points & $<12.5+$ & $12.5-40$ points & $42.5-57.5$ points & $>57.5$ points \\
\hline PUCAI & Drop of $\geq 20$ points & $<10$ points & 10-34 points & 35-64 points & $\geq 65$ points \\
\hline
\end{tabular}

Partial list of references used for this table: [refs 192031 ].

${ }^{*}$ PCDAl remission was originally set at $<10$ points ${ }^{31}$; a more accurate definition is a composite of $<10$ points or $<7.5$ without the height item. ${ }^{19}$

tSensitivity and specificity $94 \% / 93 \%$ by the Physician Global Assessment, ${ }^{20} 58 \% / 84 \%$ by mucosal healing. ${ }^{31}$

PCDAl, Pediatric Crohn's Disease Activity Index; PUCAI, Pediatric Ulcerative Colitis Activity Index; wPCDAl, weighted PCDAI. 
instruments (eg, IMPACT) and therefore should be captured concurrently.

Regardless of the choice of the endpoint, steroid/EEN-free complete remission (whether clinical or endoscopic) is preferred and should be sustained over time in maintenance of remission studies. Clinical endpoints and PROs should be combined with indirect serum and/or faecal biomarkers of inflammation.

\section{ULCERATIVE COLITIS}

The most widely used endoscopic scoring system in children is the Mayo subscore of $0-3$ points. Other available scores include the UCEIS, ${ }^{23}$ the Ulcerative Colitis Colonoscopic Index of Severity ${ }^{24}$ and the Modified Mayo Endoscopic Score (MMES); see details in the recent guidelines on endoscopic evaluation in PIBD from ESPGHAN's IBD Porto group. ${ }^{25}$ The MMES captures the severity of inflammation in the entire colon by adding the Mayo subscores in each of the colonic segments. The expected added benefit has been recently found to be limited in adults ${ }^{26}$ although this might differ in children who have more often pancolitis. Nonetheless, the theoretical benefit probably does not justify the large increment of invasiveness associated with a complete colonoscopy as compared with limited sigmoidoscopy. Most investigators now agree that an endoscopic subscore of 0 should be regarded as mucosal healing but a score of 1 may be considered in selected trials based on the intervention under study and the eligible baseline severity. Correlation between macroscopic and microscopic inflammation is good for Mayo 0 but not for Mayo 1, and histological remission is associated with improved long-term outcomes. ${ }^{27}$ Histological scoring, however, cannot currently be used as a major endpoint measure because of lack of validation and limited reliability but could be considered as a secondary endpoint to support mucosal healing.

The high concordance of the PUCAI with mucosal inflammation, described above, has led the EMA but not the FDA to recommend it as a primary endpoint when endoscopic assessment is not required and for assessing disease activity at interim visits without endoscopic assessment (http://www.ema.europa. eu/docs/en_GB/document_library/Regulatory_and_procedural_ guideline/2016/01/WC500200026.pdf).

Several other measures are important as secondary endpoints in paediatric UC. The IMPACT-III questionnaire is validated as a disease-specific measure of health-related quality of life for children $\geq 9$ years of age. ${ }^{28}$ Faecal calprotectin is an important secondary outcome measure especially in visits when endoscopic assessment is not performed. ${ }^{29}$ However, given the large variability of results and the lack of a validated cut-off for mucosal healing, faecal markers alone cannot be used as primary endpoints. The development of a paediatric PRO for paediatric UC (TUMMY-UC) is underway under the qualification programme of both the FDA and the EMA and will likely be incorporated in future clinical trials.

The primary and secondary outcomes are best determined by the agent under study. For drugs that have extensive adult data on mucosal healing and are not first in class, endoscopic evaluation should not be a primary outcome. Paediatric-specific disease activity scores such as PUCAI should be used as a primary outcome supplemented by faecal calprotectin. Eliminating the endoscopic procedure would significantly facilitate recruitment. All pre-registration trials of drugs of a new category, however, should require steroid-free mucosal healing as their primary outcome. Endoscopic evaluation would be required at 8-12 weeks for induction trials and at 54 weeks for maintenance.
Maintenance of remission trials should span at least 1 year in order to allow adequate time to assess relapses. A primary endpoint of relapse-free and steroid-free sustained clinical remission at both week 30 and week 54 is recommended, with an endoscopic evaluation at week 54 to assess for mucosal healing. ${ }^{22}$

Growth is rarely impaired in UC and thus not an important outcome measure. Some degree of osteopenia is seen in $20 \%-30 \%$ of children with UC, much less often than in CD.

\section{CROHN'S DISEASE}

Multi-item measures of disease activity, a concept incorporating symptoms, signs and biomarkers, have, until recently, constituted the primary endpoint in CD clinical trials. The PCDAI or its derivatives have been most often used in children ${ }^{19} 30$ (tables 3 and 4). Of the shortened PCDAI versions, the wPCDAI best maintains validity, while demonstrating greater feasibility and responsiveness to short-term change, as needed in induction trials. ${ }^{20}$ Both versions however have only fair correlations with endoscopic appearance judged by the SES-CD $(r=0.33-0.45){ }^{31}$

Given the discrepancy between symptoms and presence of active intestinal inflammation, assessment of mucosal healing via complete ileocolonoscopy has emerged as an important clinical trial endpoint also in paediatric CD trials. The performance characteristics of the most commonly used endoscopic indices, Crohn's Disease Endoscopic Index of Severity (CDEIS), ${ }^{32}$ its simpler derivative, the SES-CD, ${ }^{33}$ and the Rutgeerts postoperative endoscopic score, were recently critically evaluated in adults. ${ }^{34}$ Mucosal healing should be defined as SES-CD 0-2 points or CDEIS $<6$ points. Endoscopic response is defined as at least $50 \%$ reduction of baseline measure if performed but this may not be available in children when the baseline ileocolonoscopy is not mandated.

The use of ileocolonoscopies for research purposes in children should be judicious given its invasiveness and need for general anaesthesia and bowel cleansing. Thus, at most one follow-up ileocolonoscopy for any given paediatric trial may be reasonable, and preferably for drugs representing new categories.

Recently, the MINI index has been developed to reflect mucosal healing at study visits that do not include endoscopic assessment. ${ }^{21}$ It is weighted on faecal calprotectin and includes also CRP, ESR and the stooling item from the PCDAI. The sensitivity/specificity of a MINI $<8$ to reflect mucosal healing (ie, SES-CD $<3$ points) was $86 \% / 83 \%$ (AUROC 0.92 , 95\% CI 0.89 to $0.96 ; \mathrm{p}<0.001)$; and $95 \%$ of those with a score $<8$ had at most mild inflammation. Although promising, more data are necessary to evaluate its utility in clinical practice and trials.

Ileocolonoscopic examination cannot assess the proximal small intestine (L $4 \mathrm{~b}$ disease), as occurs in 10\%-15\% of children. In addition, although the cecum is reached in over $90 \%$ of colonoscopies performed in paediatric CD, the ileum may not be intubated in up to $20 \%-25 \%$ of cases $(20 \%$ in the ImageKids study $[n=240$ children $]$ and $26 \%$ in the Eurokids registry $[n=1227]) .{ }^{35}$ In the North American RISK inception cohort, however, lower failure rates have been reported $(n=1176$; personal communications). Imputing the ileal subscore is mandatory to avoid biasing the results towards the milder cases, as it has been demonstrated that children without ileal intubation have more inflamed ileum and right colon. To impute the ileum by MRE: if the MaRIA score of the ileum is zero impute the ileal SES-CD as zero; otherwise

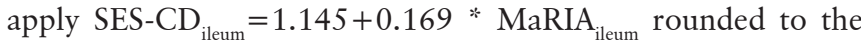
nearest whole number. Using this imputation resulted in a more accurate classification of mucosal healing. In the prospective ImageKids multicentre study, multi-item measures of intestinal 
inflammation (Pediatric Inflammatory Crohn's MRE Index) and of damage (pMEDIC) are being developed and validated. ${ }^{36}$

Growth and bone health are impaired in 30\%-50\% of children with CD so measures of both should be incorporated as independent outcome measures. ${ }^{37}$ For patients who have not completed puberty, height velocity standardised for bone age is the most sensitive measure of linear growth. However, $\geq 6$ months' interval is required between measures, precluding growth as an endpoint in short-term induction trials.

Despite numerous studies, no validated and ideal cut-off value for normal faecal calprotectin for use in IBD studies has been reported. In our previously published guideline on the management of Pediatric Ulcerative Colitis (part 1), ${ }^{12}$ we explored existing evidence and recommended cut-off values $<100 \mu \mathrm{g} / \mathrm{g}$ to reflect remission and values $>250 \mu \mathrm{g} / \mathrm{g}$ for mucosal inflammation. The authors of a recent systematic review ${ }^{38}$ suggest calprotectin $<250 \mu \mathrm{g} / \mathrm{g}$ to indicate absence of inflammation and values $>500 \mu \mathrm{g} / \mathrm{g}$ for mucosal ulceration. Further confirmatory studies are required before this can be definitively recommended.

\section{HOW TO OPTIMISE ENROLMENT: SPECIFICATIONS AND PITFALLS}

\section{Statements}

1. To enhance enrolment to PIBD trials $(97 \%$ agreement $)$ :

A. Set a realistic sample size for children.

B. Ensure that all children with moderate- severe disease can be rapidly treated with an effective medication, thus minimising screening period to at most 3-5 working days, while relying mostly on local investigations.

C. Minimise washout periods from previous drugs.

D. Minimise repeated invasive tests-make an effort to mirror routine practice as much as possible.

E. Avoid placebo and suboptimal care.

F. Minimise time interval between the adult and paediatric trials.

Several factors make recruitment into paediatric trials more difficult than adult trials:

- The number of prevalent IBD children is less than one tenth of the number of adult patients. The increasing number of novel drugs in the research pipeline means more competition for recruitment into trials involving the same limited paediatric population.

- Parents are frequently concerned about potential adverse events and are hesitant to perform invasive procedures.

- Paediatric trials often begin years after the drugs have received adult approval thus are available to children offlabel outside the trial. Two industry-initiated paediatric trials have recently failed to meet recruitment milestones and terminated prematurely. The major issue in one study was the inclusion of placebo but the other was merely an unattractive study design unrelated to placebo.

A feasible trial is one that mirrors routine practice as much as possible. In addition to the aforementioned potential barriers, a major preventable pitfall is avoiding a long screening period. A typical study design in biologics may require moderate-severe disease both at screening and at randomisation. Adding the time to effect of the study drug, the period in which the child's disease is active becomes unbearable and unethical. Screening period should ideally not surpass $\sim 3-4$ working days, relying on local labs for required tests (eg, blood tests, pregnancy, stool cultures and tuberculosis screening), and using the central labs only as a post-randomisation verification. Site readers of endoscopic results are more likely to generate higher scores than the central readers, influencing results of clinical trials. ${ }^{39}$ However, central reading should not postpone randomisation and thus sponsors must ensure turnaround time of no more than 48 hours for paediatric trials. If this is not possible, then unlike in adults, central reading should be used to verify local reading; in case of a mismatch between the local and the central readers, the randomised child may be rapidly withdrawn or be enrolled in a separate open-label arm.

Similarly, the washout period from previous drugs must be realistic. Asking, for instance, 8 weeks' washout from previous biologics will exclude most children who poorly tolerate active disease. Individualising the washout period by measuring trough levels and demonstrating undetectable levels can help overcome this limitation. Minimising number of study visits and connecting via telephone or home visit may also increase recruitment.

Increasing participants' awareness of the health problem being studied, and its potential impact on their health, can increase recruitment to clinical studies. ${ }^{40}$

Nonetheless, even the best designed study should not have an overly ambitious recruitment goal. Sample sizes should be kept to the minimum that is needed to satisfy scientific rationale, using available evidence from every trial participant. Without addressing study-specific power calculations, as a very general rule a realistic sample size for a complicated paediatric RCT may be $60-120$ children while an $\sim 200$ targets may be still feasible for a very simple trial that mimics clinical practice. Sample size may be affected by the availability of prior convincing data from adults, choice and objectivity of endpoints, use of biomarkers demonstrating target engagement and whether the drug is first in class. Consideration should be given to use of adaptive trial designs and approaches such as modelling/simulation, which may minimise uncertainties in assumptions of data extrapolation. ${ }^{41}$

\section{FINAL NOTE}

This paper is aimed at all trials of drug efficacy and safety. Recruitment may prove to be unrealistic if a study is too burdensome, poses uncertainty or leaves the child with active disease for more than several days without an effective treatment. Long washout and screening periods, use of placebo, multiple study visits, colonoscopies and venipunctures are all immediate barriers for enrolment. We hope the viewpoints expressed in this paper will assist planning RCTs which are both of high quality and ethical, while being feasible in PIBDSupplementary file 1 .

\section{Author affiliations}

${ }^{1}$ Pediatric Gastroenterology Institute, Shaare Zedek Medical Center, The Hebrew University of Jerusalem, Jerusalem, Israel

${ }^{2}$ Division of Gastroenterology, Hepatology and Nutrition, Department of Paediatrics,

The Hospital for Sick Children, University of Toronto, Toronto, Ontario, Canada

${ }^{3}$ Child Life and Health, University of Edinburgh and Department of Paediatric

Gastroenterology and Nutrition, Royal Hospital for Sick Children, Edinburgh, UK

${ }^{4}$ Projections Research, Phoenixville, Pennsylvania, USA

${ }^{5}$ Children's Hospital of Philadelphia, University of Pennsylvania, Philadelphia

Pennsylvania, USA

${ }^{6}$ Department of Paediatric Gastroenterology and Nutrition, Royal Hospital for Children, Glasgow, Scotland, UK

${ }^{7}$ Department of Pediatrics, Susan and Leonard Feinstein IBD Center, Icahn School of Medicine, Mount Sinai, New York, USA

${ }^{8}$ Department of Paediatrics, University of California San Francisco, San Francisco,

California, USA

${ }^{9}$ Department of Paediatric Gastroenterology, Sophia Children's Hospital/ Erasmus MC University, Rotterdam, The Netherlands

${ }^{10}$ Connecticut Children's Medical Center, Hartford, Connecticut, USA

${ }^{11}$ Department of Paediatric Gastroenterology, Hepatology and Nutrition, Hospital

Sant Joan de Déu, Barcelona, Spain

${ }^{12}$ Children's National Health System, Washington, DC, USA

${ }^{13}$ ReveraGen BioPharma, LLC, Rockville, MD, USA 
${ }^{14}$ Division of Gastroenterology and Hepatology, Mayo Clinic, Rochester, Minnesota, USA

${ }^{15}$ Division of Gastroenterology and Hepatology, Dr v Hauner Children's Hospital, LMU Munich, Munich, Germany

${ }^{16}$ Division of Gastroenterology, Hepatology, and Nutrition, Department of Medicine, Boston Children's Hospital, Boston, Massachusetts, USA

${ }^{17}$ Université Paris-Descartes, Paris-Sorbonne Cité, Paris, France

${ }^{18}$ Assistance Publique-Hopitaux de Paris, Hôpital Necker Enfants Malades, Service de Gastroentérologie pédiatrique, Paris, France

${ }^{19}$ INSTITUT IMAGINE - INSERM 1163, Paris, France

Acknowledgements RKR is part funded by a NRS Senior Fellowship and whose work is currently supported by a grant from The Leona M and Harry B Helmsley Charitable Trust and the Catherine Mcewan Foundation.

Contributors All authors contributed equally, and read and approved the final submitted version.

Funding The authors have not declared a specific grant for this research from any funding agency in the public, commercial or not-for-profit sectors.

Competing interests DT received consultation fees, research grant, royalties, or honorarium from Janssen, Pfizer, Hospital for Sick Children, Ferring, AstraZeneca, AbbVie, Takeda, Boehringer Ingelheim, Biogen, Atlantic Health, Shire, Celgene and Lilly for the last 3 years. AMG received consultation fees, research grant or honorarium from AbbVie, Celgene, Ferring, Gilead, Janssen, Lilly, Pfizer, Shire and Takeda for the last 3 years. DW received consultation fees, honoraria, research support or meeting expenses from AbbVie, Takeda, Ferring, Falk, Napp, Predictimmune and Roche for the last 3 years. LdR received consultation fees, honoraria or meeting expenses from AbbVie, Celltrion, Shire, Pfizer, Mallinckrodt and Nestlé for the last 3 years. DRM is president of a consulting company that provides service to the pharmaceutical industry. RKR received speaker fees and travel support and participated in medical board meetings with AbbVie, Janssen, Shire, Celltrion, NAPP and Nestle. MBH received research grant support from Genentech, AbbVie, Janssen, Takeda, Sucampo and Shire; is a member of the advisory board for Gilead and Genentech; received education grant from Mallinckrodt. FMR received speaker fees, consultation fees, research grants or honorarium from Janssen, AbbVie, Centocor, MSD France, Nestlé Nutrition Institute, Nestlé Health Science, Danone, Mead Johnson; Takeda, Celgene, Biogen, Shire, Pfizer, Therakos and ARKOPHARMA for the last 3 years. LC received honoraria and travel fees from Janssen for the last 3 years; is an employee of ReveraGen BioPharma and owns stock options in ReveraGen. JH is a member of the advisory board for Janssen and AbbVie; is a consultant of Pfizer, Roche, Lilly, Receptos and Boehringer Ingelheim. AB received research support from Prometheus, Janssen, AbbVie, Takeda and Buhlmann; consulting fees from Shire, Takeda and Best Doctors; and honoraria/royalties from Up to Date, Boston University and Nutricia. RNB is a member of the pediatric advisory board for AbbVie, Janssen, Pfizer and Celgene. MD received consultant fees or research support from Janssen, Abbvie, Roche, UCB, Pfizer, Takeda, Arena, Prometheus, Celgene and Lilly for the last three years. JMdC received consultation fees, honoraria or meeting expenses from AbbVie, Celltrion, MSD Spain, Dr Falk, Abbott, Lactalis, Otsuka, Roche, Celgene and Nestlé for the last 3 years. SK received consultant fees or research support from AbbVie, Berlin-Chemie, Biocodex, BioGaia, Danone, Mead Johnson, Nestle Nutrition, Shire and Thermo Fisher for the last 3 years.

Patient consent for publication Obtained.

Provenance and peer review Not commissioned; externally peer reviewed.

\section{ORCID iD}

Frank M Ruemmele http://orcid.org/0000-0001-5571-4957

\section{REFERENCES}

1 Kelsen JR, Grossman AB, Pauly-Hubbard H, et al. Infliximab therapy in pediatric patients 7 years of age and younger. J Pediatr Gastroentero/ Nutr 2014;59:758-62.

2 Adedokun OJ, Xu Z, Padgett L, et al. Pharmacokinetics of infliximab in children with moderate-to-severe ulcerative colitis: results from a randomized, multicenter, openlabel, phase 3 study. Inflamm Bowel Dis 2013;19:2753-62.

3 Ruemmele FM, Veres G, Kolho KL, et al. Consensus guidelines of ECCO/ESPGHAN on the medical management of pediatric Crohn's disease. J Crohns Colitis 2014:8:1179-207.

4 Hyams JS, Chan D, Adedokun OJ, et al. Subcutaneous golimumab in pediatric ulcerative colitis: pharmacokinetics and clinical benefit. Inflamm Bowel Dis 2017;23:2227-37.

5 Dunne J, Rodriguez WJ, Murphy MD, et al. Extrapolation of adult data and other data in pediatric drug-development programs. Pediatrics 2011;128:e1242-e1249.

6 de Ridder L, Assa A, Bronsky J, et al. Use of biosimilars in pediatric inflammatory bowel disease: an updated position statement of the pediatric IBD Porto Group of ESPGHAN. J Pediatr Gastroenterol Nutr 2019;68:144-153.
7 Barbour AM, Fossler MJ, Barrett J. Practical considerations for dose selection in pediatric patients to ensure target exposure requirements. Aaps J 2014;16:749-55.

8 Feber J, Al-Matrafi J, Farhadi E, et al. Prednisone dosing per body weight or body surface area in children with nephrotic syndrome: is it equivalent? Pediatr Nephrol 2009:24:1027-31.

9 Adedokun OJ, Xu Z, Marano CW, et al. Pharmacokinetics and exposure-response relationship of golimumab in patients with moderately-to-severely active ulcerative colitis: results from Phase 2/3 PURSUIT induction and maintenance studies. J Crohns Colitis 2017;11:35-46.

10 Mulugeta Y, Barrett JS, Nelson R, et al. Exposure matching for extrapolation of efficacy in pediatric drug development. J Clin Pharmacol 2016;56:1326-34.

$11 \mathrm{Xu} \mathrm{Z}$, Mould DR, Hu C, et al. Population pharmacokinetic analysis of infliximab in pediatrics using integrated data from six clinical trials. Clin Pharmacol Drug Dev 2012;1:203.

12 Turner D, Ruemmele FM, Orlanski-Meyer $\mathrm{E}$, et al. Management of paediatric ulcerative colitis, part 1: ambulatory care-an evidence-based guideline From European crohn's and colitis organization and european society of paediatric gastroenterology, hepatology and nutrition. J Pediatr Gastroenterol Nutr 2018:67:257-91

$13 \mathrm{Xu} \mathrm{Z}$, Davis HM, Zhou H. Rational development and utilization of antibody-based therapeutic proteins in pediatrics. Pharmacol Ther 2013;137:225-47.

14 Turner D, Koletzko S, Griffiths AM, et al. Use of Placebo in Pediatric Inflammatory Bowel Diseases: A Position Paper From ESPGHAN, ECCO, PIBDnet, and the Canadian Children IBD Network. J Pediatr Gastroenterol Nutr 2016:62:183-7.

15 Hueber W, Sands BE, Lewitzky S, et al. Secukinumab, a human anti-IL-17A monoclonal antibody, for moderate to severe Crohn's disease: unexpected results of a randomised, double-blind placebo-controlled trial. Gut 2012;61:1693-700.

16 Birimberg-Schwartz L, Zucker DM, Akriv A, et al. Development and validation of diagnostic criteria for ibd subtypes including ibd-unclassified in children: a multicentre study from the pediatric IBD Porto group of ESPGHAN. J Crohns Colitis 2017; 11:1078-1084.

17 Turner D, Griffiths AM, Veerman G, et al. Endoscopic and clinical variables that predict sustained remission in children with ulcerative colitis treated with infliximab. Clin Gastroenterol Hepatol 2013;11:1460-5

18 Turner D, Seow CH, Greenberg GR, et al. A systematic prospective comparison of noninvasive disease activity indices in ulcerative colitis. Clin Gastroenterol Hepatol 2009;7:1081-8.

19 Turner D, Griffiths AM, Walters TD, et al. Appraisal of the pediatric Crohn's disease activity index on four prospectively collected datasets: recommended cutoff values and clinimetric properties. Am J Gastroenterol 2010;105:2085-92.

20 Turner D, Griffiths AM, Walters TD, et al. Mathematical weighting of the pediatric Crohn's disease activity index (PCDAI) and comparison with its other short versions. Inflamm Bowel Dis 2012;18:55-62.

21 Ben Shoham A, Cozijnsen MA, Kang B, et al. Development and validation of the "min index" (mucosal inflammation non-invasively index) for crohne"s disease on a large prospective pediatric cohort. J Ped Gastroenterol Nutr 2017;65(Suppl 1):S5.

22 Ruemmele FM, Hyams JS, Otley A, et al. Outcome measures for clinical trials in paediatric IBD: an evidence-based, expert-driven practical statement paper of the paediatric ECCO committee. Gut 2015;64:438-46.

23 Arai M, Naganuma M, Sugimoto S, et al. The ulcerative colitis endoscopic index of severity is useful to predict medium- to long-term prognosis in ulcerative colitis patients with clinical remission. J Crohns Colitis 2016;10:1303-9.

24 Samuel S, Bruining DH, Loftus EV, et al. Validation of the ulcerative colitis colonoscopic index of severity and its correlation with disease activity measures. Clin Gastroenterol Hepatol 2013;11:49-54.

25 Oliva S, Thomson M, de Ridder L, et al. Endoscopy in pediatric inflammatory bowel disease: a position paper on behalf of the porto IBD Group of the European society for pediatric gastroenterology, hepatology and nutrition. J Pediatr Gastroenterol Nutr 2018;67:414-30.

26 Ferrante M, Verstockt B, Gijbels L, et al. Su1831 - Evaluation of segmental endoscopic disease activity in ulcerative colitis through the Modified Mayo Endoscopic Score (MMES): results from a prospective study. Gastroenterology 2018;154:S-599-0.

27 Bessissow $T$, Lemmens B, Ferrante $M$, et al. Prognostic value of serologic and histologic markers on clinical relapse in ulcerative colitis patients with mucosal healing. Am J Gastroenterol 2012;107:1684-92.

28 Otley A, Smith C, Nicholas D, et al. The IMPACT questionnaire: a valid measure of health-related quality of life in pediatric inflammatory bowel disease. J Pediatr Gastroenterol Nutr 2002:35:557-63.

29 Yamaguchi S, Takeuchi Y, Arai K, et al. Fecal calprotectin is a clinically relevant biomarker of mucosal healing in patients with quiescent ulcerative colitis. $J$ Gastroenterol Hepatol 2016;31:93-8.

30 Hyams JS, Ferry GD, Mandel FS, et al. Development and validation of a pediatric Crohn's disease activity index. J Pediatr Gastroenterol Nutr 1991;12:439-47.

31 Turner D, Levine A, Walters TD, et al. Which PCDAI Version Best Reflects Intestinal Inflammation in Pediatric Crohn Disease? J Pediatr Gastroenterol Nutr 2017;64:254-60.

32 Mary JY, Modigliani R. Development and validation of an endoscopic index of the severity for Crohn's disease: a prospective multicentre study. Groupe d'Etudes 
Thérapeutiques des Affections Inflammatoires du Tube Digestif (GETAID). Gut 1989;30:983-9.

33 Daperno M, D'Haens G, Van Assche G, et al. Development and validation of a new, simplified endoscopic activity score for Crohn's disease: the SES-CD. Gastrointest Endosc 2004;60:505-12.

34 Vuitton L, Marteau P, Sandborn WJ, et al. IOIBD technical review on endoscopic indices for Crohn's disease clinical trials. Gut 2016;65:1447-55.

35 de Bie $\mathrm{Cl}$, Buderus S, Sandhu BK, et al. Diagnostic workup of paediatric patients with inflammatory bowel disease in Europe: results of a 5 -year audit of the EUROKIDS registry. J Pediatr Gastroenterol Nutr 2012;54:374-80.

36 Turner D, Gavish M, Focht G, et al. Development of the Pediatric Inflammatory Crohn's MRE Index (PICMI)- results from the ImageKids Study. ECCO annual meeting; Feb 16th 2018, Vienna, 2018.

37 Gasparetto M, Guariso G. Crohn's disease and growth deficiency in children and adolescents. World J Gastroenterol 2014;20:13219-33.

38 Heida A, Park KT, van Rheenen PF. Clinical utility of fecal calprotectin monitoring in asymptomatic patients with inflammatory bowel disease: a systematic review and practical guide. Inflamm Bowel Dis 2017;23:894-902.

39 Feagan BG, Sandborn WJ, D'Haens G, et al. The role of centralized reading of endoscopy in a randomized controlled trial of mesalamine for ulcerative colitis. Gastroenterology 2013;145:149-57.

40 Caldwell PH, Hamilton S, Tan A, et al. Strategies for increasing recruitment to randomised controlled trials: systematic review. PLoS Med 2010;7:e1000368.

41 Kelly LE, Dyson MP, Butcher NJ, et al. Considerations for adaptive design in pediatric clinical trials: study protocol for a systematic review, mixed-methods study, and integrated knowledge translation plan. Trials 2018;19:572.

42 Markowitz J, Grancher K, Kohn N, et al. A multicenter trial of 6-mercaptopurine and prednisone in children with newly diagnosed Crohn's disease. Gastroenterology 2000;119:895-902.

43 Escher JC. European Collaborative Research Group on Budesonide in Paediatric IBD. Budesonide versus prednisolone for the treatment of active Crohn's disease in children: a randomized, double-blind, controlled, multicentre trial. Eur J Gastroenterol Hepatol 2004;16:47-54.

44 Bousvaros A, Guandalini S, Baldassano RN, et al. A randomized, double-blind trial of Lactobacillus $\mathrm{GG}$ versus placebo in addition to standard maintenance therapy for children with Crohn's disease. Inflamm Bowel Dis 2005;11:833-9.

45 Hyams J, Crandall W, Kugathasan S, et al. Induction and maintenance infliximab therapy for the treatment of moderate-to-severe Crohn's disease in children. Gastroenterology 2007;132:863-73.

46 Hyams JS, Griffiths A, Markowitz J, et al. Safety and efficacy of adalimumab for moderate to severe Crohn's disease in children. Gastroenterology 2012:143:365-74

47 Lazzerini M, Martelossi S, Magazzù G, et al. Effect of thalidomide on clinical remission in children and adolescents with refractory Crohn disease: a randomized clinical trial. JAMA 2013;310:2164-73.

48 Ferry GD, Kirschner BS, Grand RJ, et al. Olsalazine versus sulfasalazine in mild to moderate childhood ulcerative colitis: results of the Pediatric Gastroenterology Collaborative Research Group Clinical Trial. J Pediatr Gastroenterol Nutr 1993:17:32-8.

49 Hyams J, Damaraju L, Blank M, et al. Induction and maintenance therapy with infliximab for children with moderate to severe ulcerative colitis. Clin Gastroenterol Hepatol 2012;10:391-9.

50 Winter HS, Krzeski P, Heyman MB, et al. High- and low-dose oral delayed-release mesalamine in children with mild-to-moderately active ulcerative colitis. J Pediatr Gastroenterol Nutr 2014:59:767-72.

51 Lazzerini M, Martelossi S, Magazzù G, et al. Effect of thalidomide on clinical remission in children and adolescents with ulcerative colitis refractory to other immunosuppressives: pilot randomized clinical trial. Inflamm Bowel Dis 2015:21:1739-49.

52 Turner D, Vlamakis H, Marcus D, et al. Manipulating the microbiome in pediatric Acute Severe Colitis with a cocktail of antibiotics: a pilot randomized controlled trialPRASCO. ESPGHAN annual meeting, Geneva, 2018. 